\title{
Dissolution Highlights from the 2012 AAPS Annual Meeting in Chicago
}

\author{
Nikoletta Fotaki ${ }^{1}$,*, Gregory P. Martin², and Johannes Krämer ${ }^{3}$ \\ ${ }^{1}$ University of Bath, UK \\ ${ }^{2}$ Complectors Consulting, Pottstown, PA, USA \\ ${ }^{3}$ PHAST, Germany
}

e-mail:n.fotaki@bath.ac.uk

T he 2012 AAPS Annual Meeting provided several programs on dissolution and related topics, including two symposia on biorelevant testing, a roundtable on gelatin capsule issues, and the business meeting of the In Vitro Release and Dissolution Testing Focus Group (IVRDT FG). There was also a workshop on the topic of oral bioperformance, which is reported on elsewhere in this issue of Dissolution Technologies.

\section{PREDICTING IN VIVO ORAL DRUG ABSORPTION USING BIO-RELEVANT IN VITRO TESTING: CURRENT APPROACHES AND FUTURE DIRECTIONS}

"Predicting In Vivo Oral Drug Absorption using Biorelevant In Vitro Testing: Current Approaches and Future Directions" was discussed in a symposium. David Vodak from Bend Research talked about in vitro tests for reliable selection of active amorphous forms and solid dosage forms. After discussing principles of in vitro testing and types of amorphous dispersions, he described the common models of disintegration and erosion and the link of the disintegration and dissolution rates to absorption. He highlighted the importance of the mechanistic understanding of the dissolution process and presented two case studies in which microcentrifuge methods were used for definition of precipitate, formulation selection, and dissolution advantage of amorphous systems. Ping Gao from Abbot Laboratories talked about a biphasic system combining the flow cell with USP Apparatus 2 with dual paddle for selection and optimization of formulations of low-solubility compounds. He pointed out the importance of biorelevant tests for the assessment of supersaturation and precipitation kinetics of the drug. A case study with the proposed biphasic method for formulations of a BCS Class 2 compound and the development of in vitro-in vivo (animal and human data) relationship was presented. Amitava Mitra from Merck talked about the use of biorelevant dissolution and absorption modeling for optimization of enabled formulations. The principles of absorption modeling were discussed, and case studies of the dissolution of weak base, crystalline nanosuspensions and micronized and nanosized formulation of BCS Class 4 compounds in combination with absorption modeling were presented. Neil Mathias from Bristol-Myers Squibb discussed the dissolution of weakly basic drugs and

${ }^{*}$ Corresponding author. described situations that lead to elevated gastric $\mathrm{pH}$. The current in vivo (dog, monkey, and rat) and in vitro (Apparatus 2, Apparatus 4, minidissolution) models used to assess $\mathrm{pH}$ were noted, and a new in vitro, $\mathrm{pH}$-effect model (microdissolution system with UV fiber-optic probe) with emphasis on kinetics of dissolution, supersaturation, and precipitation was described. The application of this model for the assessment of the risk of $\mathrm{pH}$ effect was pointed out. Several case studies were presented, and the challenges of this method in terms of dose impact, undissolved material, and turbidity were discussed.

\section{BIORELEVANT IN VITRO PERFORMANCE TESTING OF ORALLY ADMINISTERED DOSAGE FORMS}

The Symposium on Biorelevant In Vitro Performance Testing of Orally Administered Dosage Forms proved to be one of the most popular presentations of the Annual Meeting. The first presentation, "Bio-relevant Performance Testing, An Overview" by Christos Reppas of the National and Kapodistrian University of Athens, reviewed the physiology of the GI tract and showed how luminal composition varies dramatically by region. He pointed out that imaging technologies and in vivo sampling, while possible, are costly and challenging, and that traditional solubility studies are not good at predicting the actual solubility in the lumen. Use of second-generation biorelevant media, such as FaSSGF-V2 and FeSSIF-V2, may be better at predicting in vivo dissolution than earlier versions. Ultimately, there are still many questions about whether in vitro tests using biorelevant media really are useful and how we can improve our understanding of in vivo transport mechanisms.

David Sperry of Eli Lilly gave the second presentation, "The Changing Gastrointestinal Environment and Its Influence on Dosage Form Performance." Echoing some of the concepts from the first speaker, he focused on the dynamic nature of the $\mathrm{Gl}$ tract and the important role of agitation, which traditional dissolution testing does not mimic well. He suggested the use of alternatives such as the Dynamic Gastric Model, which is designed to simulate gastric function more effectively; the TIM gastrointestinal models; or the homemade Artificial Stomach Device (ASD) as tools for product development. He presented case studies in which the ASD was useful for just this purpose and concluded that the dynamic nature of the Gl tract may require labs to use a spectrum of devices from com- 
pendial apparatus to the ASD to the TIM to best understand product performance.

The complexity of the factors affecting product performance was again broached in the third talk, "Integration of Biorelevant Dissolution Data with PBPK Models during Formulation Development," presented by Filippos Kesisoglou from Merck. He showed that combining dissolution data with physiologically based pharmacokinetic (PBPK) modeling may provide a link to PK data and guide dissolution studies. While in vitro-in vivo correlation (IVIVC) remains the "gold standard," it is not easy to accomplish, particularly in early development. Solubility studies, in silico investigations such as Gastro Plus or DDD Plus, and dissolution studies on drug substance may be useful, and complex dissolution studies are not always required for formulation selection at early stages. As biorelevant dissolution models continue to evolve, he recommends incorporation of computational models with appropriate in vitro tests.

Sandra Sharp from FDA concluded the symposium with a very interesting talk titled "Setting Clinically Relevant Dissolution Specifications: A Regulatory Perspective." Starting from the premise that clinically relevant specifications (CRS) take into consideration the clinical impact of variations in critical quality attributes and process parameters, there is the potential for manufacturing changes to be linked with meaningful in vitro data to enable the development of science- and risk-based specifications. Approaches for establishing CRS may include range established based on (1) batches tested in pivotal phase 3 clinical trials, (2) a range of release characteristics resulting in bioequivalence, or 3 ) predictive and robust in vivo in vitro correlations. She concluded that "FDA encourages the conduct of $\mathrm{BA} / \mathrm{BE}$ studies during drug product development to establish the relationship between drug product variants with different release characteristics and plasma levels [that may lead to] establishment of clinically relevant drug product specifications, potential for developing IVIVC models, potential for wider drug product specifications, and regulatory flexibility within the QbD framework."

The symposium demonstrated that there is significant interest in exploring dissolution testing using biorelevant conditions, which may be useful for product development, and that if clinically relevant specifications are developed (as described by the FDA), there is the potential for regulatory flexibility.

\section{DISSOLUTION AND DISINTEGRATION METHODS FOR GELATIN CAPSULES}

The topic Dissolution and Disintegration Methods for Gelatin Capsules, including Dietary Supplements was discussed in a roundtable session. Vivian Gray from V. A. Gray Consulting presented analytical issues related to enzyme addition when cross-linking occurs. She described the new text in USP $<711>$ in the subsec- tion regarding dosage forms containing or coated with gelatin and pointed out that there is no similar section in the European and Japanese Pharmacopoeia. She highlighted that enzyme methods are used only when failure occurs in a study, and she then talked about the new method for determining pepsin activity and emphasized incompatibility issues of enzymes in media containing surfactant that may require a pre-soak step. She discussed the topics described in General Chapter $<1094>$, such as cross-linking causes, dissolution process development, and validation by clearly defining the three-stage dissolution process (rupture of the shell, release and dispersion of the capsule fill material, and dissolution of the active ingredient). Raimar Löbenberg from the University of Alberta discussed the differences between pharmaceuticals and dietary supplements in USP Performance Testing. After describing the quality attributes for capsules, he talked about the disintegration test as described in USP General Chapter $<701>$. He highlighted the aspects of the disintegration test and the influence of the beaker specifications on the results as revealed by a study performed. Then he presented a case study in which differences between the disintegration and rupture test results were observed for the same products. Dominique Cade from Capsugel presented issues relating to capsule testing in nutraceuticals. He described the two disintegration apparatus and discussed the relevant test for hard gelatin capsules and the impact of the capsule size on the results. He emphasized the advantages of the disintegration test with automated end point and discussed the applicability of this method to cross-linking capsules. A lively discussion moderated by Bob Chapman from Midwestern University followed the three short presentations. Some questions were related to the use of a higher amount of enzyme for stability studies and the position of regulatory authorities on this approach. The lack of international harmonization on the use of enzymes was highlighted during the discussion. Several questions regarding the activity and the amount of enzyme were posed. Testing the activity of the enzyme before each experiment, checking the $\mathrm{pH}$, and adding an amount of enzyme based on the level of crosslinking were suggested. The insufficiency of enzyme characterization methods for GMP requirements due to lack of reproducibility was also pointed out. Questions regarding the physiological relevance of the enzyme concentration were raised. The differences between the quality control approach and the physiological relevance approach were noted. It was mentioned that results from scintigraphic studies have revealed that even though the fill of the capsule empties in the stomach, the body of the capsule does not break. The applicability of the stress device for the in vitro testing of the capsules was stated. Discussion of the effects of cross-linking on bioavailability took place, and the differences between the characteristics of natural cross-linking and the forced 
cross-linking under accelerated conditions by formaldehyde were reported. Applicability of the procedure to chewable capsules and the suitability of the medicated gum apparatus for this purpose were also discussed.

\section{IVRDT FOCUS GROUP FACE-TO-FACE MEETING}

The annual face-to-face business meeting of the In Vitro Release and Dissolution Testing Focus Group was held at midday on Tuesday. In a presentation by outgoing chair Alger Salt (GSK) and incoming chair Greg Martin (Complectors Consulting), the accomplishments and activities for 2012 were reviewed and goals and objectives for 2013 presented. The focus group currently has over $800 \mathrm{mem}-$ bers, most of whom are regular members of AAPS but over $20 \%$ of whom are students. The significant interest of students in joining this group will influence its activities in 2013, with attempts to meet the needs of this constituency. The focus group is affiliated with three sections of AAPS: Analysis and Pharmaceutical Quality Section (APQ), Formulation Development and Design (FDD), and Regulatory Support (RS). Those interested in learning more or joining the focus group can visit https://www.aaps.org/ In_Vitro_Release_and_Dissolution_Testing/. This website is interactive with a Discussion Board. Recent presentations will also be available on the website.

The Focus Group continued to be active in 2012. Xujin Lu was elected to the Chair-Elect position. We continued collaboration with the Nutraceutical and Natural Products Chemistry Focus Group, presenting the symposium Dissolution and Disintegration Methods for Gelatin Capsules, Including Dietary Supplements. The group organized and gained approval for an international outreach program workshop to be held in Argentina in 2013 and developed program proposals for the 2013 annual meeting (of which two will be presented, Biorelevant In Vitro Dissolution Testing of Non-Oral Dosage Forms and Emerging Dissolution Technologies). Members of the focus group collaborated on an article on media selection to be published in

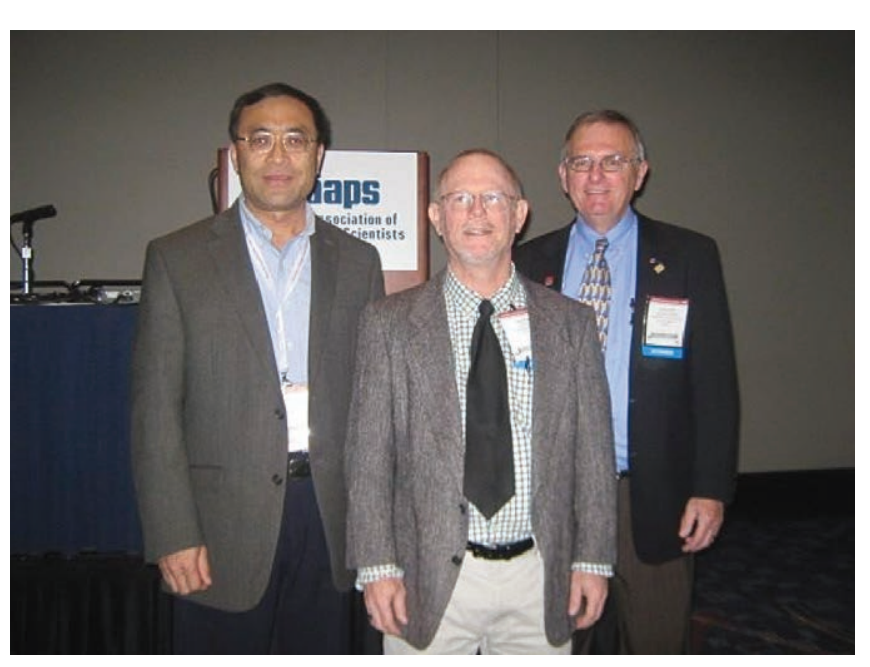

In Vitro Release and Dissolution Focus Group Officers from left to right: Chair-Elect, Xujin Lu; Past Chair, Alger Salt; and Chair, Greg Martin.

Dissolution Technologies and are working on a series of webinars to be presented in 2013, starting with Investigation of Dissolution Changes and Failures, to be followed by The Role of Dissolution Testing Across the Life Cycle of a Pharmaceutical Product. Focus group members also are contributing to a Special Edition on Automation in the May, 2013 issue of Dissolution Technologies.

The activities of the focus group will continue logically into 2013 with the Outreach Program in Argentina and presentation of the webinars and programming. In addition, ways to increase interaction with students and to promote publications in the field of dissolution testing will be pursued. The Focus Group continues to provide opportunities for those interested in dissolution testing to communicate and to exchange information about developments in the field. 